\title{
Covid-19 risks and response in South Asia
}

\author{
The region is ill prepared for the crisis that lies ahead
}

\author{
Zulfiqar A Bhutta professor ${ }^{1}$, Buddha Basnyat professor ${ }^{2}$, Samir Saha professor ${ }^{3}$, Ramanan \\ Laxminarayan professor ${ }^{4}$
}

${ }^{1}$ Center of Excellence in Women and Child Health, Aga Khan University, Karachi, Pakistan ; ${ }^{2}$ Oxford University Clinical Research Unit-Patan Academy of Health Sciences, Kathmandu, Nepal; ${ }^{3}$ Child Health Research Foundation, Dhaka, Bangladesh; ${ }^{4}$ Center for Disease Dynamic, Economics and Policy, New Delhi, India

Over the past few weeks we have seen political leaders, policy makers, and health managers grapple with the reality of a novel coronavirus outbreak and its potential for global spread. ${ }^{12}$ From what initially seemed like a localised outbreak in Hubei province of China in December 2019, it rapidly became clear that SARS-CoV-2 had pandemic potential. ${ }^{3}$ But it was almost two months before the World Health Organization declared a true global pandemic. The number of deaths from covid-19 in Italy now exceeds those reported from China, and the outbreak in Iran may have seeded cases in Pakistan and Afghanistan. ${ }^{4}$

The number of reported cases in South Asia remains relatively low, however, and the response patchy. By 24 March, authorities in the South Asian Association for Regional Cooperation (SAARC), which comprises India, Pakistan, Bangladesh, Nepal, Sri Lanka, Maldives, Bhutan, and Afghanistan, had reported just 1536 confirmed cases and 22 deaths. ${ }^{5}$ Given extremely limited diagnostic testing, the region may not have sufficient information to gauge the true extent of the epidemic and is ill prepared for the potential crisis that lies ahead. Although all countries took individual actions, it took the collective political leadership of SAARC almost two months to put aside political differences and meet to discuss the dangers to the region posed by covid- $19 .^{6}$

There is as yet no indication of a joint response, however, and the actions of individual countries in SAARC have varied. The kind of lockdown and mass social distancing that is being enforced in parts of Europe and North America may be difficult to implement in the region since most services and people may be unable to work remotely or from home. Although the potential economic and social consequences of such measures could be huge, allowing the counterfactual reality of continued regular activities and mass congregations would be extremely unwise.

Testing at scale is limited, focusing mainly on individuals arriving from affected countries and their immediate contacts. This approach is unlikely to be enough by itself, and preliminary mathematical models suggest that community transmission in
India and Pakistan may have begun in early March (unpublished data). The only effective intervention implemented is that all countries in the region have drastically reduced or stopped air travel and imposed quarantine protocols on people arriving by road or air from countries with covid-19 transmission. However, facilities for screening and quarantine remain limited and of questionable quality.

\section{Act in unison}

Irrespective of initial inertia and varied response, SAARC countries need to move rapidly and in unison if the region is to avoid a public health catastrophe on the scale of the influenza epidemic of 1918-19, which is conservatively estimated to have killed over 6 million people in British India alone. ${ }^{8}$ An exponential increase in testing capacity is needed. Supply of expensive imported kits is currently limited, and the approach to testing differs across all SAARC countries This must be standardised urgently and an accurate database of everyone tested (and their results) developed, maintained, and shared. The region has advanced pharmaceutical and biotechnology manufacturing platforms, so rapid development and deployment of high quality, low cost rapid diagnostic systems can and should be prioritised.

Whatever the short term political and economic costs, effective social distancing, promotion of hand hygiene, and other preventive protocols must be rapidly andstrictly implemented, with lockdowns if needed. At the time of writing, India had just started a 21 day lockdown. Capacity for isolation, personal protective equipment for healthcare workers, and importantly, facilities for critical care must be enhanced. Critical care capacity is a problem in all SAARC countries. Relatively few hospitals are able to isolate patients and provide intensive care with respiratory support. South Asia has an estimated 0.7-2.8 critical care beds per 100000 population. $^{9}$ Authorities have focused inappropriately on buying ventilators without paying adequate attention to the extra human resources (trained intensivists, nurses, and respiratory care technicians) required to run these services. 
Covid-19 has exposed glaring gaps in public health preparedness for infectious disease outbreaks in South Asia. The lack of a robust infectious disease surveillance and control system is particularly evident. For a region that has sent rockets to Mars and includes two nuclear powers, lack of a single academic centre with the epidemiological expertise to model a rapidly progressing epidemic seems remarkable, has proved to be a major limitation for evidence informed planning.

Sharing accurate information and best practices in real time is also critical to help counter the rapid spread of false and sometimes dangerous disinformation. Academics and healthcare professionals in this region of 1.9 billion people must stand together if we are to overcome probably the greatest public health challenge of our generation.

Competing interests: We have read and understood BMJ policy on declaration of interests and have no interests to declare.

1 Wang C, Horby PW, Hayden FG, Gao GF. A novel coronavirus outbreak of global health concern. Lancet 2020;395:470-3. 10.1016/S0140-6736(20)30185-9 31986257

2 Zhu N, Zhang D, Wang W, etal. China Novel Coronavirus Investigating and Research Team. China novel coronavirus investigating and research team. a novel coronavirus from patients with pneumonia in China, 2019. N Engl J Med 2020;382:727-33. 10.1056/NEJMoa2001017 31978945

3 WHO Director General. Media briefing 11 Mar 2020. https://www.who.int/dg/speeches/ detail/who-director-general-s-opening-remarks-at-the-media-briefing-on-covid-19---11march-2020

4 Tuite AR, Bogoch II, Sherbo R, Watts A, Fisman D, Khan K. Estimation of coronavirus disease 2019 (COVID-19) Burden and potential for international dissemination of infection from Iran. Ann Intern Med 2020; [Epub ahead of print]. 10.7326/M20-0696. 32176272

5 Center for Systems Science and Engineering. Coronavirus covid-19 global cases, 22 Mar 2020. https://www.arcgis.com/apps/opsdashboard/index.html\#/ bda7594740fd40299423467b48e9ecf6

6 South Asian Association for Regional Cooperation. Video conference of SAARC leaders to discuss measures to contain the spread of COVID19 in the region. Press release, 15 Mar 2020. http://saarc-sec.org/news/detail front/pres-release-the-leaders-of-the-memberstates-of-the-south-asian-association-for-regional-cooperation-saarc-held-a-videoconference-on-15-march-2020-to-discuss-measures-to-contain-the-spread-of-covid-19in-the-region.

7 Experts worried over South Asia Quarantine Centers. Gandhara. Reuters 2020 Mar 18. https://gandhara.rferl.org/a/experts-worried-over-south-asia-quarantine-centers/30495579. html

8 Spreeuwenberg $\mathrm{P}$, Kroneman M, Paget J. Reassessing the global mortality burden of the 1918 influenza pandemic. Am J Epidemiol 2018;187:2561-7.

10.1093/aje/kwy19130202996

9 Phua J, Faruq MO, Kulkarni AP, etal. Asian Analysis of Bed Capacity in Critical Care (ABC) Study Investigators, and the Asian Critical Care Clinical Trials Group. Critical care bed capacity in Asian countries and regions. Crit Care Med 2020

10.1097/CCM.0000000000004222. 31923030

Published by the BMJ Publishing Group Limited. For permission to use (where not already granted under a licence) please go to http://group.bmj.com/group/rights-licensing/ permissions 